\title{
IMMUNOEXPRESSION OF CD-10 IN BASAL CELL CARCINOMA AND SQUAMOUS CELL CARCINOMA OF HEAD AND NECK
}

\author{
Maria Ilyas, Muhammad Yasir Rafiq ${ }^{*}$, Farha Farhan**, Misbah Ali***, Saeed Afzall ${ }^{* * * *}$, Zainab Niazi***** \\ Baqai Dental College, Karachi Pakistan, ${ }^{*}$ Combined Military Hospital Malir/National University of Medical Sciences (NUMS) Pakistan, ${ }^{* * F o u n d a t i o n ~ C o l l e g e ~ o f ~}$ \\ Dentistry, Islamabad Pakistan, ${ }^{* * *}$ Margalla Institute of Health Sciences, Islamabad Pakistan, ${ }^{* * * *}$ Alicenna Medical College, Lahore Pakistan, \\ *****Islamabad Medical \& Dental College, Islamabad Pakistan
}

\begin{abstract}
Objective: To compare the expression of CD-10 in basal cell carcinoma (BCC) and squamous cell carcinoma (SCC). Study Design: Comparative cross-sectional study.

Place and Duration of Study: Armed Forces Institute of Pathology, Rawalpindi, from Jun 2016 to Jun 2017. Methodology: After taking ethics approval from the ethical review committee AFIP, previously diagnosed, thirty-eight cases of each basal cell carcinoma and squamous cell carcinoma were taken from the histopathology department of Armed Forces Institute of Pathology, Rawalpindi. CD-10 was applied according to standard protocol. The intensity of the CD-10 expression is assessed and categorized into three groups. CD-10 expression less than $10 \%$ in the tumour cell was taken as negative (-), 10 to $50 \%$ staining was taken as positive $(+)$ and greater than $50 \%$ staining in tumour cells was taken as strong positive $(++)$.

Results: Out of the total 76 cases included in the study, $9(24 \%)$ were strongly positive (++) whereas $20(52.6 \%)$ were positive $(+)$ and $9(23.7 \%)$ were negative (-) for CD-10 expression. Out of the total 38 cases of SCC 4 (11\%) cases showed positive CD-10 expression and $34(89 \%)$ showed negative $(-)$ results. A significant association ( $p$-value $<0.001)$ was seen when CD-10 expression in BCC was compared with SCC.

Conclusion: CD-10 expression was higher in basal cell carcinoma when compared to squamous cell carcinoma in which CD-10 expression was very low/absent, hence CD-10 can serve as a useful adjunct marker in differentiating between basal cell carcinoma and squamous cell carcinoma in problematic cases.
\end{abstract}

Keywords: Basal Cell Carcinoma (BCC), CD-10, Squamous cell carcinoma (SCC).

How to Cite This Article: Ilyas M, Rafiq MY, Farhan F, Ali M, Afzal S, Niazi Z. Immunoexpression of CD-10 in Basal Cell Carcinoma and Squamous Cell Carcinoma of Head and Neck. Pak Armed Forces Med J 2021; 71(6): 2175-2179. Doi: https://doi.org/10.51253/pafmj.v71i6.3495

This is an Open Access article distributed under the terms of the Creative Commons Attribution License (https://creativecommons.org/licenses/by-nc/4.0/), which permits unrestricted use, distribution, and reproduction in any medium, provided the original work is properly cited.

\section{INTRODUCTION}

Non Melanoma Skin Cancers (NMSCs) are the most commonly diagnosed cancers and the incidence continues to rise. The term NMSC includes all skin cancers that do not arise from melanocytes, but it is usually referred to the two most common keratinocytic tumours-BCC and SCC. These two tumours collectively account up to $95 \%$ of NMSCs. ${ }^{1}$ The most common site for NMSCs are head and neck and $90 \%$ of all skin cancers consist of cutaneous squamous cell carcinoma and cutaneous basal cell carcinoma collectively. ${ }^{2}$

Globally BCC is the commonest malignancy in humans. Its incidence is inversely proportional to the geographical latitude and is higher in white skinned population. In Australia, it is 4 folds more common that all the other cancers combined. SCC is second most common skin cancer. It is more common in men, elderly, sun exposed areas of the skin and in light skinned individuals. ${ }^{3}$

Correspondence: Dr Maria Ilyas, Department of Oral Pathology, Baqai Dental College, Karachi Pakistan

Received: 17 Nov 2019; revision received: 19 Apr 2020; accepted: 08 May 2020
In 2004 according to the Karachi Cancer Registry skin cancer account for $2.6 \%$ of all cancers 4 . Among the malignant cancers, skin tumours account for $8.38 \%$ of malignant tumours in males and $8.50 \%$ in females making skin tumours the second most common malignant tumour in our country. ${ }^{5}$

NMSCs being highly prevalent affects the life quality of a large number of people and additionally pose a considerable financial constraint on the country's economy and great amount of budget is spent on their diagnosis and treatment. Numerous studies have been done and it has been proven that the size of the tumour and treatment cost are directly proportional. ${ }^{6}$

CD-10 also known as common acute lymphoblastic leukemia antigen (CALLA), neutral endopeptidase (NEP), enkephalinase, and membrane metallo endopeptidase (MME). ${ }^{7}$ CD-10 is a cell surface zinc dependent metallo endopeptidase and its expression is observed in various hematopoietic, non-lymphoid as well as neoplastic cells, ${ }^{8,9} \mathrm{CD}-10$ is expressed in acute lymphoblastic leukemia, follicular lymphoma, Burkitt lymphoma and some other hematopoietic tumours. Its 
expression can also be seen in normal tissues such as lymphoid precursor cells, the brush border of enterocytes, renal tubules, glomeruli, myoepithelial cells of the breast, hair follicles, eccrine glands and sebaceous glands. ${ }^{10}$

The histological features of keratotic BCC may appear similar to well differentiated SCC and their differential diagnosis is difficult. Incorrect diagnosis in these cases may lead to unnecessary removal of additional tissue or aggressive surgical therapy.

The study was conducted to compare the expression of CD-10 in BCC and SCC specimens which may prove helpful in distinguishing between these two entities. If an association was observed, it would aid in the differential diagnosis of histologically overlapping SSC and BCC cases. Moreover, it would be helpful in early and correct diagnosis, predicting patient's outcome and prognosis. The outcome of the study would be helpful in early and correct diagnosis which is directly related to the prognosis, and can be used in improving and customizing the management of the tumour.

\section{METHODOLOGY}

This comparative cross sectional study was carried out on total 76 cases, 38 of BCC and 38 of SCC, each collected from Histopathology Department at Armed Forces Institute of Pathology, Rawalpindi, from June 2016 to June 2017. Ethical Approval from the Institutional Ethical Review Committee was taken. Using the WHO sample calculator, keeping the confidence level at $95 \%$, and anticipated population proportion $(\mathrm{P})$ at 0.87 and margin of error at $5 \%$ a sample size was calculated.

Inclusion Criteria: Paraffin embedded blocks of previously and freshly diagnosed cases of SCC and BCC of patients of all ages and both genders were included in the study.

Exclusion Criteria: Necrotic, scarce, autolysed and cases that had previously received chemotherapy, radiotherapy or treatment were excluded.

The blocks were cut and serial sections of $4 \mu \mathrm{m}$ were prepared. The sections were stained with haemotoxylin and eosin. All biopsies were reviewed again by a senior pathologist with subspecialty interest in oral cancers and diagnosis were confirmed.

CD-10 antibody was applied according the standard protocol. Haematoxylin was applied as a counterstain and sections mounted with coverslip for light microscopy.
The intensity of the expression of CD-10 in the lesional cells is assessed according to the Sabeti et al.'s criteria. ${ }^{10}$ Cytoplasmic \pm staining was considered positive. The expression of CD 10 staining was categorized into three groups: 1 ) less than $10 \%$ tumour cells positive $=$ negative $(-),(2) 10-50 \%$ positive cell=positive $(+)$, 3) more than $50 \%$ positive cells $=$ strong positive $(++)$. For statistical analysis purpose, cases having positive $(+)$ and strong positive $(++)$ expression were considered as positive and negative (-) were considered as negative.

The information collected in the form of variables was analysed using Statistical Package for Social Sciences (SPSS) version 21. For quantitative variables such as age mean, Standard Deviation was calculated. For qualitative variables such as gender, site of biopsy and histological variant, frequency and percentages were calculated. Expression of CD-10 was compared by applying Chi square test was used to compare the scores of CD-10 in patients with BCC and SCC and p-value of $\leq 0.05$ was considered significant.

\section{RESULTS}

This study included a total of 76 cases, 38 each of SCC and BCC. Out of the total 38 cases of BCC included in our study the mean age of patients was 63.18 \pm 14.41 years. Among the BCC cases $27(71.05 \%)$ were male whereas $11(28.94 \%)$ patients were female. The highest proportion of tumours was observed on the nose $17(44.74 \%)$. The second most common site was eye $9(23.68 \%)$ cases, $6(15.79 \%)$ cases were from the cheek and the remainder $5(13.16 \%)$ were from neck, scalp and forehead. Total 38 cases of BCC were included in the study, out of which $9(24 \%)$ were strongly positive $(++)$ whereas $20(52.6 \%)$ were positive $(+)$ and 9 (23.7 \%) were negative (-) for CD-10. Positive expression of BCC is shown in Figure-1 \& 2.

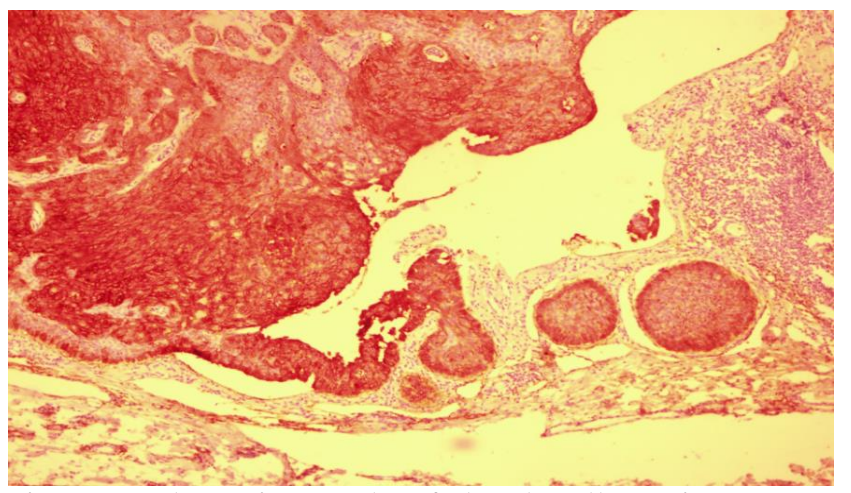

Figure-1: Photomicrograph of basal cell carcinoma nests showing strong positivity for CD 10 (CD 10 staining: 10 x10). 
Out of the total 38 cases of SCC were included in our study. Mean age of patients with SCC was $62.5 \pm$ 19.73 years. There were $24(63.16 \%)$ were male and 14 $(38.84 \%)$ were female. The lip was the most common sit for SCC accounting for $12(31.58 \%)$ cases followed by cheek accounting for $8(21.05 \%)$ cases, 7 (18.42\%) cases were on the forehead and (4) $0.53 \%$ cases were located on the nose. The remainder $7(18.42 \%)$ cases were scattered on the ear, eye neck and scalp. Out of the total 38 cases of SCC $4(11 \%)$ cases showed positive CD 10 expression and $34(89 \%)$ showed negative (-) results as shown in Figure-3.

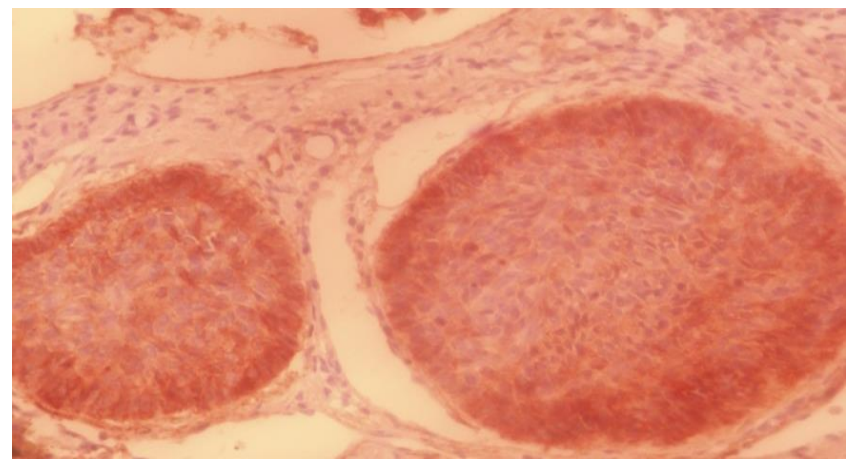

Figure-2: Photomicrograph showing strong positive cytoplasmic/membranous staining of CD-10 in BCC tumour cells (CD 10 staining: 10x40).

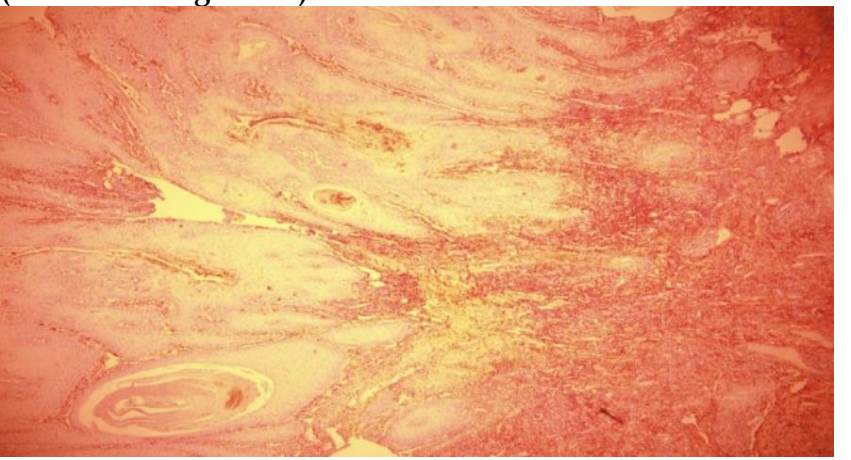

Figure-3: Photomicrograph showing negative expression of CD-10 immunostaining of SCC tumour cells and positive expression in surrounding stroma (CD-10 staining: 10x10).

There was strong associa-tion found between the CD-10 expression in basal cell carcinoma and squamous cell carcinoma $p<0.001$ shown in the Table.

Table: Association of CD-10 expression in basal cell carcinoma and squamous cell carcinoma.

\begin{tabular}{l|c|c|c}
\hline \multirow{2}{*}{ Tumour Type } & \multicolumn{2}{|c|}{ CD-10 } & \multirow{2}{*}{$\begin{array}{c}p- \\
\text { value }\end{array}$} \\
\cline { 2 - 3 } & Positive & Negative & \multirow{2}{*}{$<0.001$} \\
\hline Basal Cell Carcinoma & $29(76.3 \%)$ & $9(23.7 \%)$ & \multirow{2}{*}{ Squamous Cell Carcinoma } \\
\hline
\end{tabular}

\section{DISCUSSION}

Basal cell Carcinoma and SCC are the most common NMSCS and affect the life quality of a large number of population. They pose a considerable financial constraint on the country's economy and great amount of budget is spent on their diagnosis and treatment.

High expression of this marker indicates worse prognosis like breast carcinoma, malignant melanoma, cutaneous basal cell carcinoma and squamous cell carcinoma CD-10 in dentigerous cyst is seen in the upper layers of the epidermis. In malignant odontogenic tumour such as ameloblastoma the star shaped stellate cells show cytoplasmic reactivity whereas epithelial cells show both reactivity. Higher expression of this marker in a dentigerous cyst depicts its neoplastic nature whereas in an ameloblastoma its expression indicates locally invasiveness and high recurrence. ${ }^{11}$

BCC and SCC are biologically different but they have a similar clinical presentation. Although in most cases distinction can be made on routine hematoxylin and eosin microscopy but occasionally in superficial, fragmented or very small biopsies it is difficult to differentiate between SCC and BCC.12 Being common clinical entities and overlapping histological and clinical feature their distinction can be difficult both clinically and histologically. Differentiating between BCC and SCC is critical because of their difference in aggressiveness, metastatic potential and treatment modalities. BCC is locally destructive and usually has low metastatic potential whereas SCC has high potential for invasion associated with increased rate of fatality. SCC recurrence rate is two folds greater than BCC. Metastasis is seen in at least in $2 \%$ of SCC and it is very rare in BCC. 13

In present study, BCC was more common in the older age group. The mean age for patients was 63.18 \pm 14.41 years. Out of the total 38 cases, $27(71.05 \%)$ cases were from patients older than 50 years. 11 cases $(28.94 \%)$ were from the $33-50$ years age range and none of the cases were from the first three decades of life. Peak incidence was seen in the eight decade of life. There was a definite predilection of BCC in older age groups.

Similarly, according to a study conducted in Turkey by Koyuncuer et al ${ }^{2}$ BCC also had a predilection for the older age group. The mean age for patients with BCC was $67.2 \pm 13.9$ years. The median age in men is 67.3 years and in women was 67 years. The mean age of patients with SCC was $62.5 \pm 19.73$ years. The occurrence ages ranged 5-95 years. Out of the total 38 cases, $33(86.84 \%)$ cases were from patients who are 50 years and above and the remainder 5 (13.16\%) cases 
are below the age of 50 years. Highest incidence was seen in the sixth decade of life.

In the current study out of the total 38 cases of BCC was most common on the nose accounting for $17(44.74 \%)$ cases. Eyes were the second common site with $9(23.68 \%)$ cases on this location. $6(15.79 \%)$ cases are from the cheek and $5(13.16 \%)$ cases were from the neck, scalp and forehead collectively. Similarly, Asif et $a l$, reported that nose and the cheek were the most common location for BCC in the head and neck region. ${ }^{14}$ For SCC the lip was the common location 12 (31.58\%) cases. Second most common location for SCC is cheek 8 cases $(21.05 \%)$ followed by forehead on which 7 $(18.42 \%)$ cases were found.

Out of the total patients with SCC, 24 (63.16\%) were male and $14(38.84 \%)$ were female. The male to female ratio was 1.7:1. Among the BCC cases 11 (28.94\%) patients are female whereas $27(71.05 \%)$ were male.

Local studies determining the comparative expression of CD 10 in BCC and SCC are scarce. However there are some international studies published which have been published. For instance Sabeti et al, carried out a study on the usefulness of CD-10 in distinguishing between BCC and SCC. Out of the total 27 cases of BCC included, $6(22.22 \%)$ were strong positive, 14 (51.85\%) were positive for CD 10 expression. In contrast, out of the 17 SCC cases $15(88.24 \%)$ showed no reactivity. They proposed that $\mathrm{CD} 10$ can be used a useful adjunct marker to differentiate between SCC and BCC. They also suggested that as CD-10 shows positive staining for the inner root sheath, hair matrix, and perifollicular fibrous sheath it also supports the hypothesis that BCC maybe of follicular derivation. ${ }^{10}$

In another study, Aiad et al, ${ }^{21}$ cases of BCC were stained with CD-10 and evaluated. Seven (33.33\%) cases of BCC showed strong positive expression while $3(14.28 \%)$ showed positive expression. Out of the total 16 SCC cases, none $(0 \%)$ of the lesional cells showed any reactivity for $C D-10$. They suggested that the differential expression of CD 10 in BCC and SCC indicates that they were biologically different. Due to the structural similarity of CD-10 to MMPs it created a microenvironment that help the cancer cells to invade and metastasize. Therefore they proposed that if tum-our cells are CD-10 positive, then our diagnosis should be in favour of BCC rather than SCC. ${ }^{15}$

A similar trend was seen in a study Aslani et al, gave their analysis by comparing CD-10 expression in cutaneous BCC and SCC and between BCC and Tri- choepithelioma. Forty two (76.36\%) cases showed positive reaction with CD-10 and 13 (23.63\%) were negative. None of SCC cases included in their study showed any reactivity in the tumour cells. They also suggested that CD-10 was a useful marker when differentiating between BCC and SCC. ${ }^{12}$

Study carried out by Mazaher Ramezani et al, had a similar trend in CD-10 expression where EMA, CEA, CD-10 and BCL-2 were applied on BCC and SCC of the skin. ${ }^{16}$ Shafaei et al, reported similar trend and concluded that CD-10 can help to differentiate between SCC and BCC-17 Gaballah et al, and Heidrapour et $a l$, also supported the use of CD-10 as a differential marker for BCC and SCC. 18,19

Concordant with these findings, CD 10 was positive in $76.3 \%$ BCCs in the present study and $89 \%$ of SCC were negative. Therefore, CD 10 positive cells in the tumour nests favours the diagnosis of $\mathrm{BCC}$ over SCC and CD 10 can become a reliable adjunct marker to differentiate between BCC and SCC in problematic and superficial fragmented biopsies.

\section{LIMITATION OF THE STUDY}

In order to decrease the bias and to generalize the results to entire population, a larger sample size should be studied. However owing to a limited availability of samples and the time constraint a large sample size could not be selected.

\section{CONCLUSION}

CD-10 expression was higher in BCC when compared to SCC in which CD-10 expression was very low/absent. Hence, CD-10 can serve as a useful adjunct marker in differentiating between BCC and SCC.

\section{Conflict of Interest: None.}

\section{Authors' Contribution}

MI: Conception of study, design of the work, literature search, data collection, interpretation of the data, MYR: Data collection, revising the work critically, FF: Study design, data collection, MA: Interpretation of data analysis and statistical analysis, SA: Revising the work ciritically, final approval, supervisng the research, ZN: Data collection, data analysis.

\section{REFERENCES}

1. Bartoš V, Kullová M. Non-melanoma skin cancer-a clinicopathological study of patients with basal cell carcinoma and squamous cell carcinoma. Klin Onkol 2017; 31(1): 40-45.

2. Koyuncuer A. Histopathological evaluation of non-melanoma skin cancer. World J Surg Oncol 2014; 12(1): 159.

3. Elder DE, Massi D, Willemze R, Scolyer R. who classification of skin tumours: international agency for research on Cancer; $4^{\text {th }}$ Edition, Volume 11; 2018.

4. Jaffar N, Kehar SI, Iqbal J, Ghani R, Hasan SM. Morphological spectrum of non melanoma skin cancer experience at a tertiary care hospital in Karachi, Pakistan. Pak J Med Dent 2019; 8(1): 7-10. 
5. Jamal S, Moghal S, Mamoon N. The pattern of malignant tumours: tumour registry data analysis, AFIP, Rawalpindi, Pakistan. J Pak Med Assoc 2006; 56(8): 359-362.

6. Mudigonda T, Pearce DJ, Yentzer BA, Williford PM, Feldman SR. The economic impact of non melanoma skin cancer: a review. J Natl Comp Canc Netw 2010; 8(8): 888-896.

7. Hormozi E, Fard VN, Naseri MA. Comparison of immunohistochemical expression of CD-10 in keratocystic odontogenic tumour and ameloblastoma. Dent Res J 2016; 13(2): 110.

8. Petre N, Vrapciu AD, Rusu MC. Molecular anatomy of CD-10. J Contemp Clin Pract 2015; 1(1): 22-29.

9. Bajwa A, Khadim M, Din H, Ali SS, Jamil U, Khan UAS. Immunohistochemical expression of CD-10, BCL6 and MUM1 in differentiating diffuse large B Cell lymphoma subtypes. J Coll Physicians Surg Pak 2017; 27(1): 621-624.

10. Sabeti S, Malekzad F, Neishaboori N, Pourabdollah TM, Bidarizerehpoosh $\mathrm{F}$. The usefulness of CD-10 in distinguishing between cutaneous basal cell carcinoma and squamous cell carcinoma. Iran J Pathol 2014; 9(4): 245-250.

11. Anjum R, Naseem N, Saif R, Nagi AH. Expression of CD-10 in dentigerous cysts and ameloblastomas-a reliable prognostica-tor. Pak Oral Dent J 2015; 35(3): 404-407.

12. Aslani FS. Value of CD-10 expression in differentiating cutaneous basal from squamous cell carcinomas and basal cell carcinoma from trichoepithelioma. Iran J Med Sci 2013; 38(2): 100.
13. Heidrapour M, Rajabi P, Emami M. CD-10 Immunoreactivity in cutaneous squamous and basal cell carcinoma. Pak J Med Sci 2012; 28(3): 496-500.

14. Asif M, Mamoon N, Ali Z, Akhtar F. Epidemiological and excision margin status of basal cell carcinoma-three years armed forces institute of pathology experience in Pakistan. Asian Pac J Cancer Prev 2010; 11(5): 1421-1423.

15. Aiad HA, Hanout HM. Immunohistochemical expression of CD-10 in cutaneous basal and squamous cell carcinomas. J Egypt Natl Canc Inst 2007; 19(1): 195-201.

16. Ramezani M, Mohamadzaheri E, Khazaei S, Najafi F, VaisiRaygani A, Rahbar M, et al. Comparison of EMA, CEA, CD-10 and BCL-2 biomarkers by immunohistochemistry in squamous cell carcinoma and basal cell carcinoma of the skin. Asian Pac J Cancer Prev 2016; 17(3): 1379-1383.

17. Shafaei S, Sharifian M, Hajian-Tilaki K. Immunohistochemical expression of CD-10 in cutaneous basal and squamous cell carcinomas. Caspian J Intern Med 2015; 6(2): 103.

18. Gaballah MA, Ahmed RA. Diagnostic value of CD-10 and Bcl2 expression in distinguishing cutaneous basal cell carcinoma from squamous cell carcinoma and seborrheic keratosis. Pathol Res Pract 2015; 211(12): 931-938.

19. Heidarpour M, Rajabi P, Sajadi F. CD-10 expression helps to differentiate basal cell carcinoma from trichoepithelioma. J Res Med Sci 2011; 16(7): 938. 